\title{
Weakly trapped submanifolds in standard static spacetimes
}

\author{
Allan G. Freitas, Henrique F. de Lima, Eraldo A. Lima Jr. and Marcio S. Santos
}

\begin{abstract}
We study weakly trapped submanifolds of codimension two in a standard static spacetime. In this setting, we apply some generalized maximum principles in order to investigate the geometry of these trapped submanifolds. For instance, we establish sufficient conditions to guarantee that such a spacelike submanifold must be a hypersurface of the Riemannian base of the ambient spacetime. As a consequence, we prove that there do not exist $n$-dimensional compact (without boundary) trapped submanifolds immersed in an $(n+2)$-dimensional standard static spacetime. Such a nonexistence result was originally obtained for stationary spacetimes by Mars and Senovilla [20]. Furthermore, we also investigate parabolic weakly trapped submanifolds immersed in a standard static spacetime.
\end{abstract}

\section{Introduction}

Standard static spacetimes are examples of stationary spacetimes. Let us recall here that a stationary spacetime is a time-orientable Lorentzian manifold $\left(\bar{M}^{n+2}, \bar{g}\right)$ where there exists an infinitesimal symmetry given by a timelike Killing vector field $K$ (cf. [26]). The existence of this vector field $K$ enables us to define around each point a coordinate system $\left(x_{1}, \ldots, x_{n+1}, t\right)$ such that $K$ coincides with the coordinate vector field $\partial / \partial t$ on its domain of definition and such that the components of the metric tensor in these coordinates are independent of $t$. By normalizing $K$, we obtain an observers vector field $Z=K / \sqrt{-\bar{g}(K, K)}$. These observers measure a metric tensor that does not change with time. Furthermore, if this timelike Killing vector field is also irrotational (that is, the distribution $K^{\perp}$ of all smooth vector fields on $\bar{M}^{n+2}$ that are orthogonal to $K$ is involutive), then a local warped prod-

Key words and phrases: standard static spacetimes, weakly trapped submanifolds, parabolic spacelike submanifolds, mean curvature vector field.

2010 Mathematics Subject Classification: primary 53C42; secondary 53B30, 53C50. 
uct structure appears and the spacetime is called static (for more details see, for instance, [2]). When this structure is global this spacetime is known as a standard static spacetime. More precisely, a standard static spacetime $\left(\bar{M}^{n+2}, \bar{g}\right)$ endowed with a globally defined timelike Killing vector field $K$ is isometric to the following warped product

$$
\left(M^{n+1} \times{ }_{\rho} \mathbb{R}_{1}, \pi_{M}^{*}(\tilde{g})+\left(\rho \circ \pi_{M}\right)^{2} \pi_{\mathbb{R}}^{*}\left(-d t^{2}\right)\right),
$$

where $\pi_{M}$ and $\pi_{\mathbb{R}}$ denote the canonical projections from $M^{n+1} \times \mathbb{R}_{1}$ onto each factor, $\tilde{g}$ is the Riemannian metric on the Riemannian base $M^{n+1}, \mathbb{R}_{1}$ is the manifold $\mathbb{R}$ endowed with the metric $-d t^{2}$ and $\rho=\sqrt{-\bar{g}(K, K)}$ is the warping function. In this context, it is known that any static spacetime is locally isometric to a standard static one (cf. Proposition 12.38 of [23]). Conversely, Sánchez in [28] and more recently Aledo, Romero and Rubio in [2] obtained some sufficient conditions for a static spacetime to be standard. Other properties on the geometry of standard static spacetimes were studied by Sánchez in [27]-[29]. The importance of standard static spacetimes also comes from the fact that they include some classical spacetimes, such as the $(n+2)$-dimensional Lorentz-Minkowski space $\mathbb{L}^{n+2}$, Einstein static universe as well as models that describe an universe where there is only a spherically symmetric non-rotating mass, as a star or a black hole, like exterior Schwarzschild spacetime and some regions of Reissner-Nordström spacetime (see, for example, [8], [17]).

On the other hand, in recent decades there has been much interest in the study of spacelike submanifolds immersed in a Lorentzian manifold. Into this branch, the trapped submanifolds appear as an important particular case. We recall that a spacelike submanifold of a spacetime is said to be trapped if its mean curvature vector is timelike. The concept of trapped submanifold was first introduced by Penrose [24] in order to study singularities of a spacetime, giving rise to some of the famous singularity theorems (see [17], [18], [30]). In General Relativity, a trapped surface is a two-dimensional imbedded spatial surface such that the product of the traces of their two future-directed null second fundamental forms is everywhere positive (see [19], [31]).

The limiting case of the trapped submanifolds are the marginally trapped submanifolds, which are defined as being spacelike submanifolds whose mean curvature vector field is lightlike. We note that marginally trapped submanifolds can also be regarded as the Lorentzian dual of minimal submanifolds, when compared with the Riemannian context. For a thorough discussion concerning marginally trapped submanifolds, we refer the readers to the works [4]-[6], [10], [11].

In [16], Flores, Haesen and Ortega obtained some existence and non-existence results on trapped and marginally trapped surfaces in 4-dimensional Friedmann- 
Lemaître-Robertson-Walker (FLRW) spacetimes by using a relation between these surfaces and constant mean curvature surfaces in 3-manifolds. In particular, they showed the existence of closed marginally trapped surfaces with any genus in closed FLRW spacetimes. More recently, Alías, Cánovas and Colares [3] obtained rigidity results for codimension two marginally trapped submanifolds in a generalized Robertson-Walker (GRW) spacetime. They also derived some interesting nonexistence results for weakly trapped submanifolds (that is, the mean curvature vector field is supposed to be causal) in GRW spacetimes. Afterwards, Chruściel, Galloway and Ling [12] established a set of natural conditions which guarantee the non-existence of weakly trapped surfaces in asymptotically de Sitter spacetimes.

Here, our purpose is study the geometry of a weakly trapped submanifold $\Sigma^{n}$ of codimension two in a standard static spacetime $M^{n+1} \times{ }_{\rho} \mathbb{R}_{1}$. In this setting, we establish sufficient conditions to guarantee that $\Sigma^{n}$ must be a hypersurface of the Riemannian base $M^{n+1}$. As a direct consequence, we conclude that there do not exist compact (without boundary) codimension 2 trapped submanifolds immersed in a standard static spacetime. Such a nonexistence result was originally obtained for stationary spacetimes by Mars and Senovilla [20] (see also [32]). Furthermore, we also investigate parabolic weakly trapped submanifolds immersed in such a spacetime (see Section 4). In order to obtain our results, we apply a suitable formula for the Laplacian of the height function naturally attached to $\Sigma^{n}$ and a sufficient criterion to apply the generalized maximum principle of Omori [22] and Yau [35] (see Section 3). Before, in Section 2 we recall some basic facts concerning weakly trapped submanifolds immersed in a standard static spacetime.

\section{Preliminaries}

Let $\bar{M}^{n+2}$ be a $(n+2)$-dimensional Lorentzian manifold endowed with a timelike Killing vector field $K$. Suppose that the distribution $\mathcal{D}$ orthogonal to $K$ is of constant rank and integrable. We denote by $\Psi: M^{n+1} \times \mathbb{I} \rightarrow \bar{M}^{n+2}$ the flow generated by $K$, where $M^{n+1}$ is an arbitrarily fixed spacelike integral leaf of $\mathcal{D}$ labeled as $t=0$, which will be assumed connected, and $\mathbb{I}$ is the maximal interval of definition. In what follows, we will consider $\mathbb{I}=\mathbb{R}$.

In this setting, $\bar{M}^{n+2}$ can be regard as a standard static spacetime $M^{n+1} \times_{\rho} \mathbb{R}_{1}$, that is, the product manifold $M^{n+1} \times \mathbb{R}$ endowed with the warping metric

$$
\langle,\rangle=\pi_{M}^{*}\left(\langle,\rangle_{M}\right)-\left(\rho \circ \pi_{M}\right)^{2} \pi_{\mathbb{R}}^{*}\left(d t^{2}\right)
$$

where $\pi_{M}$ and $\pi_{\mathbb{R}}$ denote the canonical projections from $M \times \mathbb{R}$ onto each factor, $\langle,\rangle_{M}$ is the induced Riemannian metric on the base $M^{n+1}, \mathbb{R}_{1}$ is the manifold $\mathbb{R}$ 
endowed with the metric $-d t^{2}$ and the warping function

$$
\rho \in C^{\infty} \text { is } \rho=|K|=\sqrt{-\langle K, K\rangle},
$$

where || denotes the norm of a timelike vector field on $\bar{M}^{n+2}$.

Along this paper, we will consider a connected and oriented spacelike submanifold $\psi: \Sigma^{n} \rightarrow \bar{M}^{n+2}$ immersed in a standard static spacetime $\bar{M}^{n+2}=M^{n+1} \times{ }_{\rho} \mathbb{R}_{1}$, that is, the metric induced on $\Sigma^{n}$ via $\psi$ is a Riemannian metric. As usual, we also denote by $\langle$,$\rangle the metric on \Sigma^{n}$ induced via $\psi$. Since $K$ is a globally defined timelike vector field on $\bar{M}^{n+2}$, it follows that there exists a unitary timelike normal vector field $N$ globally defined on $\Sigma^{n}$ which is in the same time-orientation of $K$ (one can define $N$ as been the unitary direction of $K$ minus its projection on $\Sigma^{n}$ ) and, as we are assuming that $\Sigma^{n}$ is oriented, a spacelike normal vector field $\nu$ globally defined on $\Sigma^{n}$, such that $\{N, \nu\}$ constitutes an orthonormal frame for the normal bundle of $\Sigma^{n}$. By using the inverse Cauchy-Schwarz inequality, we get

$$
\langle N, K\rangle \leq-\rho<0 \quad \text { on } \quad \Sigma^{n}
$$

We call this normal vector field $N$ as been the future-pointing Gauss map of $\Sigma^{n}$. Throughout this work, $N$ will always denote the future-pointing Gauss map of a spacelike submanifold $\psi: \Sigma^{n} \rightarrow \bar{M}^{n+2}$.

It is well known that the curvature tensor $R$ of the submanifold $\Sigma^{n}$ can be described in terms of the second fundamental form $I I$ and of the curvature tensor $\bar{R}$ of the ambient spacetime $\bar{M}^{n+2}=M^{n+1} \times{ }_{\rho} \mathbb{R}_{1}$ by the Gauss equation as follows (see, for instance, [23]):

$$
\langle R(X, Y) Z, W\rangle=\langle\bar{R}(X, Y) Z, W\rangle+\langle I I(X, Z), I I(Y, W)\rangle-\langle I I(X, W), I I(Y, Z)\rangle,
$$

for every tangent vector fields $X, Y, Z, W \in \mathfrak{X}(\Sigma)$.

In this previous setting, we will consider some particular smooth functions on a connected spacelike submanifold $\psi: \Sigma^{n} \rightarrow \bar{M}^{n+2}$ immersed in a standard static spacetime $\bar{M}^{n+2}=M^{n+1} \times{ }_{\rho} \mathbb{R}_{1}$, namely, the (vertical) height function $h=\pi_{\mathbb{R}} \circ \psi$, the angle function $\Theta_{1}=\langle N, K\rangle$, where we recall that $N$ denotes the future-pointing Gauss map of $\Sigma^{n}$, and the angle function $\Theta_{2}=\langle\nu, K\rangle$.

From the decomposition $K=K^{\top}-\Theta_{1} N+\Theta_{2} \nu$, where ()$^{\top}$ denotes the tangential component of a vector field in $\mathfrak{X}(\bar{M})$ along $\Sigma^{n}$, we obtain:

$$
\nabla h=-\frac{1}{\rho^{2}} K^{\top} \quad \text { and } \quad\langle\nabla h, \nabla h\rangle=\frac{\Theta_{1}^{2}-\Theta_{2}^{2}-\rho^{2}}{\rho^{4}} .
$$


We also point out that the mean curvature vector field $\vec{H}$ along $\Sigma$ is defined by

$$
\vec{H}=-H_{N} N+H_{\nu} \nu
$$

where $H_{N}$ and $H_{\nu}$ denote the mean curvature functions in relation to the futurepointing Gauss map $N$ and the spacelike vector field $\nu$, respectively.

Remark 1 . Since the slices are totally geodesic spacelike hypersurfaces, we have that the second fundamental form of a submanifold $\psi: \Sigma^{n} \rightarrow\left\{t_{0}\right\} \times M^{n+1} \hookrightarrow \bar{M}^{n+2}$ is the lifting, via $\pi_{M}$, of the second fundamental form of the hypersurface $\tilde{\psi}=\pi_{M}$ 。 $\psi: \Sigma^{n} \rightarrow M^{n+1}$. Moreover, $N=\frac{K}{|K|}$ and $\nu$ give an orthonormal frame of the normal fiber bundle of $\psi$.

\section{Key lemmas}

In order to prove our main results in the next section, we will need some key lemmas. The first one gives a suitable formula for the Laplacian of the height function of a spacelike submanifold immersed in a standard static spacetime.

Lemma 1. Let $\Sigma^{n}$ be a spacelike submanifold immersed in a standard static spacetime $M^{n+1} \times{ }_{\rho} \mathbb{R}_{1}$. Then,

$$
\Delta h=-2\langle\nabla \ln \rho, \nabla h\rangle-\rho^{-2}\left\langle\vec{H}, \partial_{t}\right\rangle
$$

Proof. Indeed, we have that

$$
\begin{aligned}
\Delta h & =-\operatorname{div}\left(\rho^{-2} K^{\top}\right) \\
& =-\left\langle\nabla \rho^{-2}, K^{\top}\right\rangle-\rho^{-2} \operatorname{div} K^{\top} \\
& =-\left\langle\nabla \rho^{-2}, K^{\top}\right\rangle-\rho^{-2} \operatorname{div}\left(K+\Theta_{1} N-\Theta_{2} \nu\right) \\
& =\left\langle\rho^{2} \nabla \rho^{-2}, \nabla h\right\rangle-n \rho^{-2}\left(-\Theta_{1} H_{N}+\Theta_{2} H_{\nu}\right) \\
& =-\langle 2 \nabla \ln \rho, \nabla h\rangle-n \rho^{-2}\left\langle\vec{H}, \partial_{t}\right\rangle .
\end{aligned}
$$

The next key lemma gives sufficient conditions to guarantee that the Ricci curvature of a spacelike submanifold $\Sigma^{n}$ immersed in a standard static spacetime $M^{n+1} \times{ }_{\rho} \mathbb{R}_{1}$ is bounded from below. For this, we will denote by $A$ and $S$ the components of the second fundamental form of $\Sigma^{n}$ with respect to $N$ and $\nu$, respectively, that is,

$$
I I(X, Y)=\langle S X, Y\rangle \nu+\langle A X, Y\rangle N
$$


Lemma 2. Let $\bar{M}^{n+2}=M^{n+1} \times{ }_{\rho} \mathbb{R}_{1}$ be a standard static spacetime whose Riemannian base $M^{n+1}$ has nonnegative sectional curvature $K_{M}$ and convex warping function $\rho$. Let $\psi: \Sigma^{n} \rightarrow \bar{M}^{n+2}$ be a spacelike submanifold. Then,

$$
\operatorname{Ric}(X, X) \geq\left|A X+\frac{n H_{N}}{2} X\right|^{2}-\left|S X-\frac{n H_{\nu}}{2} X\right|^{2}+\varepsilon \frac{n^{2}|\vec{H}|^{2}}{4}|X|^{2},
$$

where $\varepsilon$ stands for the sign of $\langle\vec{H}, \vec{H}\rangle$.

Proof. For vector fields $U, V, W$ tangent to $\bar{M}^{n+2}$, we can write

$$
U=U^{*}+U^{\perp}
$$

where $U^{*}$ and $U^{\perp}$ are the orthogonal projections of $U$ onto $T M$ and $T \mathbb{R}_{1}$, respectively. Thus,

$$
U^{\perp}=\frac{\langle U, K\rangle}{\langle K, K\rangle} K=-\frac{\langle U, K\rangle}{\rho^{2}} K .
$$

On the other hand, with a straightforward computation it is not difficult to verify that

$$
\begin{aligned}
\bar{R}(U, V) W & =R_{M}\left(U^{*}, V^{*}\right) W^{*}+\frac{\langle V, K\rangle}{\rho^{2}} \bar{R}\left(K, U^{*}\right) W^{*}+\frac{\langle V, K\rangle\langle W, K\rangle}{\rho^{4}} \bar{R}\left(U^{*}, K\right) K \\
& -\frac{\langle U, K\rangle}{\rho^{2}} \bar{R}\left(K, V^{*}\right) W^{*}-\frac{\langle U, K\rangle\langle W, K\rangle}{\rho^{4}} \bar{R}\left(V^{*}, K\right) K
\end{aligned}
$$

Then, from Lemma 7.34 and Proposition 7.42 of [23] we get

$$
\begin{aligned}
\bar{R}(U, V) W= & R_{M}\left(U^{*}, V^{*}\right) W^{*}+\frac{\langle V, K\rangle \operatorname{Hess}_{M} \rho\left(U^{*}, W^{*}\right)}{\rho^{3}} K \\
& +\frac{\langle V, K\rangle\langle W, K\rangle\langle K, K\rangle}{\rho^{5}} \bar{\nabla}_{U^{*}} \bar{\nabla}\left(\rho \circ \pi_{M}\right) \\
& -\frac{\langle U, K\rangle \operatorname{Hess}_{M} \rho\left(V^{*}, W^{*}\right)}{\rho^{3}} K-\frac{\langle U, K\rangle\langle W, K\rangle\langle K, K\rangle}{\rho^{5}} \bar{\nabla}_{V^{*}} \bar{\nabla}\left(\rho \circ \pi_{M}\right) \\
= & R_{M}\left(U^{*}, V^{*}\right) W^{*}+\frac{\langle V, K\rangle \operatorname{Hess}_{M} \rho\left(U^{*}, W^{*}\right)}{\rho^{3}} K-\frac{\langle V, K\rangle\langle W, K\rangle}{\rho^{3}} D_{U^{*}} D \rho \\
& -\frac{\langle U, K\rangle \operatorname{Hess}_{M} \rho\left(V^{*}, W^{*}\right)}{\rho^{3}} K+\frac{\langle U, K\rangle\langle W, K\rangle}{\rho^{3}} D_{V^{*}} D \rho,
\end{aligned}
$$

where $\operatorname{Hess}_{M}$ is the Hessian on $M^{n}$. In particular, taking a local orthonormal frame $\left\{E_{1}, \ldots, E_{n}\right\}$ tangent to $\Sigma^{n}$ and $X$ a vector field tangent to $\Sigma^{n}$, we can take 
$U=W=X$ and $V=E_{i}$ in the last equation to obtain

$$
\begin{aligned}
\bar{R}\left(X, E_{i}\right) X= & R_{M}\left(X^{*}, E_{i}^{*}\right) X^{*}+\frac{\left\langle E_{i}, K\right\rangle \operatorname{Hess}_{M} \rho\left(X^{*}, X^{*}\right)}{\rho^{3}} K \\
& -\frac{\left\langle E_{i}, K\right\rangle\langle X, K\rangle}{\rho^{3}} D_{X^{*}} D \rho \\
& -\frac{\langle X, K\rangle \operatorname{Hess}_{M} \rho\left(E_{i}^{*}, X^{*}\right)}{\rho^{3}} K+\frac{\langle X, K\rangle^{2}}{\rho^{3}} D_{E_{i}^{*}} D \rho .
\end{aligned}
$$

Hence, from the last equation we conclude that

$$
\begin{aligned}
\left\langle\bar{R}\left(X, E_{i}\right) X, E_{i}\right\rangle= & \left\langle R_{M}\left(X^{*}, E_{i}^{*}\right) X^{*}, E_{i}\right\rangle+\frac{\left\langle E_{i}, K\right\rangle^{2}}{\rho^{3}} \operatorname{Hess}_{M} \rho\left(X^{*}, X^{*}\right) \\
& -\frac{\left\langle E_{i}, K\right\rangle\langle X, K\rangle}{\rho^{3}}\left\langle D_{X^{*}} D \rho, E_{i}\right\rangle-\frac{\left\langle E_{i}, K\right\rangle\langle X, K\rangle}{\rho^{3}} \operatorname{Hess}_{M} \rho\left(E_{i}^{*}, X^{*}\right) \\
& +\frac{\langle X, K\rangle^{2}}{\rho^{3}}\left\langle D_{E_{i}^{*}} D \rho, E_{i}\right\rangle \\
= & \left\langle R_{M}\left(X^{*}, E_{i}^{*}\right) X^{*}, E_{i}^{*}\right\rangle+\frac{\left\langle E_{i}, K\right\rangle^{2}}{\rho^{3}} \operatorname{Hess}_{M} \rho\left(X^{*}, X^{*}\right) \\
& -\frac{\left\langle E_{i}, K\right\rangle\langle X, K\rangle}{\rho^{3}} \operatorname{Hess}_{M} \rho\left(X^{*}, E_{i}^{*}\right) \\
& -\frac{\left\langle E_{i}, K\right\rangle\langle X, K\rangle}{\rho^{3}} \operatorname{Hess}_{M} \rho\left(X^{*}, E_{i}^{*}\right)+\frac{\langle X, K\rangle^{2}}{\rho^{3}} \operatorname{Hess}_{M} \rho\left(E_{i}^{*}, E_{i}^{*}\right) .
\end{aligned}
$$

Consequently,

$$
\begin{aligned}
\left\langle\bar{R}\left(X, E_{i}\right) X, E_{i}\right\rangle= & K_{M}\left(X^{*}, E_{i}^{*}\right)\left(\left\langle X^{*}, X^{*}\right\rangle\left\langle E_{i}^{*}, E_{i}^{*}\right\rangle-\left\langle X^{*}, E_{i}^{*}\right\rangle^{2}\right) \\
& +\frac{\left\langle E_{i}, K\right\rangle^{2}}{\rho^{3}} \operatorname{Hess}_{M} \rho\left(X^{*}, X^{*}\right) \\
& -2 \frac{\left\langle E_{i}, K\right\rangle\langle X, K\rangle}{\rho^{3}} \operatorname{Hess}_{M} \rho\left(X^{*}, E_{i}^{*}\right)+\frac{\langle X, K\rangle^{2}}{\rho^{3}} \operatorname{Hess}_{M} \rho\left(E_{i}^{*}, E_{i}^{*}\right) \\
= & K_{M}\left(X^{*}, E_{i}^{*}\right)\left(\left\langle X^{*}, X^{*}\right\rangle\left\langle E_{i}^{*}, E_{i}^{*}\right\rangle-\left\langle X^{*}, E_{i}^{*}\right\rangle^{2}\right) \\
& +\frac{1}{\rho} \operatorname{Hess}_{M} \rho\left(\widetilde{X_{i}^{*}}, \widetilde{X_{i}^{*}}\right)-\frac{2}{\rho} \operatorname{Hess}_{M} \rho\left(\widetilde{X_{i}^{*}}, \widetilde{E_{i}^{*}}\right)+\frac{1}{\rho} \operatorname{Hess}_{M} \rho\left(\widetilde{E_{i}^{*}}, \widetilde{E_{i}^{*}}\right),
\end{aligned}
$$

where $\widetilde{X_{i}^{*}}=\frac{\left\langle E_{i}, K\right\rangle}{\rho} X^{*}$ and $\widetilde{E_{i}^{*}}=\frac{\langle X, K\rangle}{\rho} E_{i}^{*}$. Hence,

$$
\begin{aligned}
\left\langle\bar{R}\left(X, E_{i}\right) X, E_{i}\right\rangle= & K_{M}\left(X^{*}, E_{i}^{*}\right)\left(\left\langle X^{*}, X^{*}\right\rangle\left\langle E_{i}^{*}, E_{i}^{*}\right\rangle-\left\langle X^{*}, E_{i}^{*}\right\rangle^{2}\right) \\
& +\frac{1}{\rho} \operatorname{Hess}_{M} \rho\left(\widetilde{X_{i}^{*}}-\widetilde{E_{i}^{*}}, \widetilde{X_{i}^{*}}-\widetilde{E_{i}^{*}}\right) .
\end{aligned}
$$


Hence, we obtain that

$$
\begin{aligned}
\sum_{i=1}^{n}\left\langle\bar{R}\left(X, E_{i}\right) X, E_{i}\right\rangle= & \sum_{i=1}^{n} K_{M}\left(X^{*}, E_{i}^{*}\right)\left(\left\langle X^{*}, X^{*}\right\rangle\left\langle E_{i}^{*}, E_{i}^{*}\right\rangle-\left\langle X^{*}, E_{i}^{*}\right\rangle^{2}\right) \\
& +\sum_{i=1}^{n} \frac{1}{\rho} \operatorname{Hess}_{M} \rho\left(\widetilde{X_{i}^{*}}-\widetilde{E_{i}^{*}}, \widetilde{X_{i}^{*}}-\widetilde{E_{i}^{*}}\right) .
\end{aligned}
$$

On the other hand, taking a local orthonormal frame $\left\{E_{1}, \ldots, E_{n}\right\}$ tangent to $\Sigma^{n}$ and using Gauss equation (2.3) jointly with (3.1), we have that the Ricci curvature Ric of $\Sigma^{n}$ is given by

$$
\begin{aligned}
\operatorname{Ric}(X, X)= & \sum_{i=1}^{n}\left\langle\bar{R}\left(X, E_{i}\right) X, E_{i}\right\rangle+\left|A X+\frac{n H_{N}}{2} X\right|^{2}-\left|S X-\frac{n H_{\nu}}{2} X\right|^{2} \\
& +\varepsilon \frac{n^{2}|\vec{H}|^{2}}{4}|X|^{2} .
\end{aligned}
$$

Therefore, since $K_{M}$ is nonnegative and $\rho$ is convex, it follows from (3.3) and (3.4) that

$$
\operatorname{Ric}(X, X) \geq\left|A X+\frac{n H_{N}}{2} X\right|^{2}-\left|S X-\frac{n H_{\nu}}{2} X\right|^{2}+\varepsilon \frac{n^{2}|\vec{H}|^{2}}{4}|X|^{2}
$$

for all tangent vector fields $X \in \mathfrak{X}(\Sigma)$.

\section{Main results}

This section is devoted to present our main results concerning the geometry of weakly trapped submanifolds immersed with codimension two in a standard static spacetime. For this reason, we will recall in the next paragraph the concept of weakly trapped submanifold (with a slight change from the one given in [3]).

A future (past) trapped submanifold is a submanifold such that the mean curvature vector $\vec{H}$ is timelike and it is future (past) pointing. A marginally future (past) trapped submanifold is a submanifold with $\vec{H}$ null, such that it is future (past) pointing. A weakly future (past) trapped submanifold is a submanifold with $\vec{H}$ causal or zero, such that it is future (past) pointing when it is causal. Finally, we recall that a minimal submanifold is a submanifold such that the mean curvature vector $\vec{H}$ is identically zero. 
In what follows, according to the nomenclature of [1], we say that a spacelike submanifold $\Sigma^{n}$ immersed in a standard static spacetime $\bar{M}^{n+2}=M^{n+1} \times_{\rho} \mathbb{R}_{1}$ is bounded away from the future infinity of $\bar{M}^{n+2}$ if there exists $\bar{t} \in \mathbb{R}$ such that

$$
\Sigma^{n} \subset\left\{(x, t) \in \bar{M}^{n+2} ; t \leq \bar{t}\right\}
$$

Analogously, we say that $\Sigma^{n}$ is bounded away from the past infinity of $\bar{M}^{n+2}$ if there exists $\underline{t} \in \mathbb{R}$ such that

$$
\Sigma^{n} \subset\left\{(x, t) \in \bar{M}^{n+2} ; t \geq \underline{t}\right\}
$$

Finally, $\Sigma^{n}$ is said bounded away from the infinity of $\bar{M}^{n+2}$ if it is both bounded away from the past and the future infinity of $\bar{M}^{n+2}$. In other words, $\Sigma^{n}$ is bounded away from the infinity if there exist $\underline{t}<\bar{t}$ such that $\Sigma^{n}$ is contained in the slab bounded by the slices $M^{n+1} \times\{\underline{t}\}$ and $M^{n+1} \times\{\bar{t}\}$.

In this setting, we obtain the following result:

Theorem 1. Let $\Sigma^{n}$ be a complete weakly future (past) trapped submanifold immersed with bounded second fundamental form in a standard static spacetime $\bar{M}^{n+2}=M^{n+1} \times{ }_{\rho} \mathbb{R}_{1}$, whose Riemannian base $M^{n+1}$ has nonnegative sectional curvature and such that the warping function $\rho$ is convex on $M^{n+1}$ outside a compact set. If $\Sigma^{n}$ is bounded away from the future (past) infinity of $\bar{M}^{n+2}$ and $\nabla \log \rho$ is bounded then either the inverse support function $\left\langle\vec{H}, \partial_{t}\right\rangle^{-1}$ or the warping function $\rho$ is unbounded.

Proof. Let $\Sigma^{n}$ be the weakly future trapped submanifold. Suppose by contradiction that $\rho$ and $\left\langle\vec{H}, \partial_{t}\right\rangle^{-1}$ are bounded on $\Sigma$. Since the second fundamental form is bounded, from Lemma 2 we have that the Ricci curvature is bounded from below. Thus, taking into account that $\Sigma^{n}$ is bounded away from the future infinity of $M^{n+1} \times{ }_{\rho} \mathbb{R}_{1}$, from Lemma 1 jointly with the generalized maximum principle of Omori [22] and Yau [35] we obtain a sequence of points $\left\{p_{k}\right\}$ in $\Sigma^{n}$ such that:

$$
0 \geq \underset{k}{\limsup } \Delta h\left(p_{k}\right)=\underset{k}{\limsup }\left(-\rho^{-2}\left\langle\vec{H}, \partial_{t}\right\rangle\right)\left(p_{k}\right) \geq 0 .
$$

Hence,

$$
\limsup _{k}\left(\left\langle\vec{H}, \partial_{t}\right\rangle\right)\left(p_{k}\right)=0
$$

Therefore $\left\langle\vec{H}, \partial_{t}\right\rangle^{-1}$ is unbounded, but it is a contradiction.

From Theorem 1 we obtain the following consequence 
Corollary 1. Let $\Sigma^{n}$ be a complete future (past) trapped submanifold immersed with bounded second fundamental form and with mean curvature bounded away from zero, that is, $|\vec{H}| \geq \alpha>0$ for some positive constant $\alpha$, in a standard static spacetime $\bar{M}^{n+2}=M^{n+1} \times{ }_{\rho} \mathbb{R}_{1}$, whose Riemannian base $M^{n+1}$ has nonnegative sectional curvature and such that the warping function $\rho$ is convex on $M^{n+1}$ outside a compact set. If $\Sigma^{n}$ is bounded away from the future (past) infinity of $\bar{M}^{n+2}$ and $\nabla \log \rho$ is bounded, then the warping function $\rho$ is unbounded.

Proof. The result follows from the proof of Theorem 1, just observing that our constraints on $\Sigma^{n}$ allow us to conclude that

$$
-\rho^{-2}\left\langle\vec{H}, \partial_{t}\right\rangle \geq \rho^{-1}|\vec{H}| \geq \rho^{-1} \alpha .
$$

Another direct consequence of Theorem 1 is the nonexistence of compact (without boundary) codimension 2 trapped submanifolds immersed in a standard static spacetime. Such a nonexistence result was originally obtained for stationary spacetimes by Mars and Senovilla [20] (see also [32]).

Corollary 2. There do not exist $n$-dimensional compact (without boundary) trapped submanifolds immersed in a standard static spacetime $\bar{M}^{n+2}=M^{n+1} \times_{\rho} \mathbb{R}_{1}$.

Proof. Since the submanifold is compact, the strong maximum principle is already granted. Therefore, we can use Lemma 1 and proceed as in the proof of Theorem 1.

Now we investigate a particular case where the above mentioned standard static spacetime $M^{n+1} \times \mathbb{R}_{1}$ is an Einstein manifold. An $(n+2)$-dimensional vacuum spacetime with cosmological constant $\Lambda$ is a Lorentzian manifold $(L, h)$ satisfying the Einstein equation $R i c=\Lambda h$. The vacuum is said to be static when $L=M^{n+1} \times{ }_{\rho} \mathbb{R}_{1}$ and $h=-\rho^{2} d t^{2}+g$, where $\left(M^{n+1}, g\right)$ is an $(n+1)$-dimensional connected Riemannian manifold, that we will take to be orientable. Furthermore, a complete and connected Riemannian manifold $\left(M^{n+1}, g\right)$ with boundary $\partial M$ (possibly empty) is said to be static if it admits a non-trivial solution $\rho \in C^{\infty}(M)$ to the equation

$$
-\left(\Delta_{g} \rho\right) g+\nabla_{g}^{2} \rho-\rho \operatorname{Ric}_{g}=0 \operatorname{in} \operatorname{int}(M) .
$$

The left hand side of equation (4.2) is the formal $L^{2}$-adjoint of the linearization of the scalar curvature operator (see [21]). We call the function $\rho$ a static potential of $M^{n+1}$. On the other hand, a solution of (4.2) in a manifold allows us to construct a spacetime satisfying the vacuum Einstein equations (with cosmological constant), whose properties, physically interpreted, justify the name static (see [13]-[15]). 
In our case, an operational advantage in dealing with these types of manifolds lies in the fact that the warping function $\rho$ satisfies an equation that relates its Laplacian with its Hessian. Taking into account (4.2), it is not difficult to verify that we can improve the last results with a weaker hypothesis of subharmonicity of the warping function $\rho$, obtaining the following:

Theorem 2. Let $\Sigma^{n}$ be a complete weakly future (past) trapped submanifold immersed with bounded second fundamental form in an Einstein standard static spacetime $\bar{M}^{n+2}=M^{n+1} \times{ }_{\rho} \mathbb{R}_{1}$, with nonnegative Ricci curvature, whose Riemannian base $M^{n+1}$ has nonnegative sectional curvature and such that the warping function $\rho$ is subharmonic in $M^{n+1}$. If $\Sigma^{n}$ is bounded away from the future (past) infinity of $\bar{M}^{n+2}$ and $\nabla \log \rho$ is bounded, then either the inverse support function $\left\langle\vec{H}, \partial_{t}\right\rangle^{-1}$ or the warping function $\rho$ is unbounded.

In order to prove the next results, we denote by $\psi=\log \rho^{2}$ the density function on $\bar{M}^{n+2}=M^{n+1} \times{ }_{\rho} \mathbb{R}_{1}$. Taking into account this background, we define the weighted Laplacian by

$$
\Delta_{\psi} u=e^{-\psi} \operatorname{div}\left(e^{\psi} \nabla u\right)
$$

where $u$ is a smooth function in $\bar{M}^{n+2}$. Let us also consider

$$
\mathcal{L}_{\psi}^{p}(\Sigma):=\left\{u: \Sigma^{n} \longrightarrow \mathbb{R}: \int_{\Sigma}|u|^{p}(x) d \mu<+\infty\right\},
$$

where $d \mu=e^{\psi(x)} d \Sigma$ stands for the weighted measure defined on $\Sigma^{n}$. Working with this setting, we obtain the following:

Theorem 3. Let $\Sigma^{n}$ be a complete weakly trapped submanifold immersed in a standard static spacetime $\bar{M}^{n+2}=M^{n+1} \times{ }_{\rho} \mathbb{R}_{1}$ and such that $|\nabla h| \in \mathcal{L}_{\psi}^{1}(\Sigma)$. Then, $\Sigma^{n}$ is a minimal spacelike submanifold. If in addition $\Sigma^{n}$ is bounded away from the infinity of $\bar{M}^{n+2}$, then $\Sigma$ is contained in a slice $M^{n+1} \times\left\{t_{0}\right\}$.

Proof. We will follow a similar structure of the proof of Proposition 2.1 of [9]. Since $\Sigma^{n}$ is complete, there exists a sequence $\phi_{j}$ such that $\phi_{j}=1$ in compact sets $K_{j} \subset \operatorname{supp} \phi_{\mathrm{j}} \subset \Omega_{j}$ which are both exhaustion of $\Sigma^{n}$. Thus, $\phi_{j} \nearrow 1$ and $\left|\nabla \phi_{j}\right|_{\infty} \rightarrow 0$ (see Proposition 4.1 of [33] for $p=1$ ). Thus,

$$
\int_{\Sigma} \operatorname{div}_{\psi}\left(\phi_{j} \nabla h\right) d \mu=\int_{\Sigma} \nabla \phi_{j} \cdot \nabla h d \mu+\int_{\Sigma} \phi_{j} \Delta_{\psi} h d \mu,
$$

where $d \mu=e^{\psi} d \Sigma$. Consequently, using the divergence theorem for the left hand side, it is equal to zero. By Holder's inequality in the first term of the right hand side, 
we obtain that it goes to 0 as $j \rightarrow \infty$. Using the monotone convergence theorem for the last term, we get

$$
0=\int_{\Sigma} \Delta_{\psi} h d \mu .
$$

Hence, since $\Delta_{\psi} h \geq 0$, we obtain that $\Delta_{\psi} h=0$. Therefore, since $\vec{H}$ is casual or zero, we must have $\vec{H} \equiv 0$.

For the second part, we observe that

$$
\Delta_{\psi} h^{2}=2 h \Delta_{\psi} h+2|\nabla h|^{2} \geq 0
$$

Since $h$ is bounded, we obtain $\left|\nabla h^{2}\right| \in \mathcal{L}_{\psi}^{1}(\Sigma)$. Thus, we can apply once more the previous argument to guarantee that $\Delta_{\psi} h^{2}=0$ and, hence, $|\nabla h|^{2}=0$ on $\Sigma^{n}$, which implies that $h$ is constant.

In the spirit of Theorem 3, we get the following result:

Corollary 3. The only compact weakly trapped submanifolds $\Sigma^{n}$ immersed in a standard static spacetime $M^{n+1} \times{ }_{\rho} \mathbb{R}_{1}$ are the minimal hypersurfaces of the slices $M^{n+1} \times\left\{t_{0}\right\}$. If in addition $\operatorname{Ric}_{M}$ is nonnegative on $M^{n+1}$ and there is a Killing vector field $Y$ on $M^{n+1}$ such that the angle between $\nu$ and $Y$ is nonnegative, then $\Sigma^{n}$ is a totally geodesic hypersurface of a slice $M^{n+1} \times\left\{t_{0}\right\}$.

Proof. The first part follows directly from Theorem 3. For the second one, we recall the following well known formula (see, for instance, equation (24) of [9])

$$
\Delta\langle\nu, Y\rangle=-\left(\left|A_{\nu}\right|^{2}+\operatorname{Ric}_{M}(\nu, \nu)\right)\langle\nu, Y\rangle
$$

Therefore, applying the divergence theorem on the above equation we get that $A_{\nu}=0$ and, hence, $\Sigma$ must be totally geodesic.

Reasoning as in the proof of Theorem 3, we also obtain the following consequence:

Corollary 4. The only $\psi$-parabolic weakly future (past) trapped submanifold $\Sigma^{n}$ immersed in a standard static spacetime $\bar{M}^{n+2}=M^{n+1} \times{ }_{\rho} \mathbb{R}_{1}$, bounded away from the future (past) infinity of $\bar{M}^{n+2}$, are the minimal hypersurfaces of the slices $M^{n+1} \times\left\{t_{0}\right\}$.

Proof. As in the proof of Theorem 3, we get that the height function $h$ is $\psi$-subharmonic and bounded from above and, therefore, it is constant. Using again the previous argument, we obtain the desired result. 
The following concept comes from the notion of probability on a manifold with a certain metric and a density not necessarily of finite integral, and it will be an important tool for the statements and proofs of the next results.

Let us consider a real function $f: \Sigma^{n} \rightarrow \mathbb{R}$ and $1 \leq p<\infty$. We define the $\psi$-weighted $p$-capacity of a compact subset $K \subset \Sigma^{n}$ as the following:

$$
\operatorname{Cap}_{\psi, p}(K)=\inf \left\{\int_{\Sigma}|\nabla \phi|^{p} d \mu ; \phi \equiv 1 \text { in } K \text { and } \phi \in C_{0}^{1}(\Sigma)\right\},
$$

where $d \mu=e^{\psi} d \Sigma$. It is natural to use the following notion of $\psi$-weighted $p$-parabolicity: a Riemannian manifold $\Sigma^{n}$ is said to be $\psi$-weighted $p$-parabolic if the $\psi$-weighted $p$-capacity of all compact subsets of $\Sigma^{n}$ is zero. With this notion in mind, it is possible to enunciate the following result as a key to prove a $p$-parabolic version for uniqueness (see Proposition 4.1 of [33]).

Proposition 1. A Riemannian manifold $\Sigma^{n}$ is $\psi$-weighted p-parabolic if, and only if, there exists a sequence of functions $\phi_{j} \in C_{0}^{1}(\Sigma)$ such that $0 \leq \phi_{j} \leq 1, \phi_{j} \nearrow 1$ uniformly on every compact subset of $\Sigma^{n}$ and

$$
\int_{\Sigma}\left|\nabla \phi_{j}\right|^{p} d \mu \longrightarrow 0
$$

Taking into account Proposition 1 and proceeding in a similar way as in the proof of Theorem 3, we obtain the following result:

Theorem 4. The only n-dimensional weakly trapped $\psi$-weighted p-parabolic submanifolds immersed in a standard static spacetime $\bar{M}^{n+2}=M^{n+1} \times{ }_{\rho} \mathbb{R}_{1}$ such that $|\nabla h| \in \mathcal{L}_{\psi}^{q}(\Sigma)$, with $\frac{1}{p}+\frac{1}{q}=1$, are the minimal spacelike submanifolds.

To close our paper, we recall that the Bakry-Émery Ricci tensor (see [7]) is defined by

$$
\operatorname{Ric}_{\psi}=\text { Ric }+ \text { Hess } \psi \text {. }
$$

In this setting, we obtain the following:

Theorem 5. Let $\Sigma^{n}$ be a complete weakly future (past) trapped submanifold immersed in the standard static spacetime $M^{n+1} \times{ }_{\rho} \mathbb{R}_{1}$, such that $h \geq 0(h \leq 0)$. If $h \in \mathcal{L}_{\psi}^{p}(\Sigma)$ with $p>1$, then $\Sigma^{n}$ is a hypersurface contained in a slice $M^{n+1} \times\left\{t_{0}\right\}$. Moreover, if the Bakry-Émery Ricci tensor of $\Sigma^{n}$ is nonnegative, $\rho$ is bounded along $\Sigma^{n}$ and $h>0(h<0)$ then $\Sigma^{n}$ is compact.

Proof. Under our constraints and taking into account that $\Delta_{\psi} h=-e^{-\psi}\left\langle\vec{H}, \partial_{t}\right\rangle$, we obtain that $h($ or $-h)$ is a nonnegative subharmonic function on $\Sigma^{n}$. Thus, since 
we are assuming that $h \in \mathcal{L}_{\psi}^{p}(\Sigma)$ with $p>1$, we can apply Theorem 1.1 of [25] to conclude that $h$ is constant on $\Sigma^{n}$, that is, $\Sigma^{n}$ is a hypersurface contained in a slice $M^{n+1} \times\left\{t_{0}\right\}$. Moreover, supposing that $h$ has strict sign, we get that $\operatorname{Vol}(\Sigma)$ is finite. Hence, if in addition the Bakry-Émery Ricci tensor of $\Sigma^{n}$ is nonnegative and $\rho$ is bounded along $\Sigma^{n}$, we can apply Theorem 1.3 of [34] to conclude that $\Sigma^{n}$ must be compact.

Acknowledgements. The authors would like to thank the referee for reading the manuscript in great detail and for his/her valuable suggestions and useful comments which improved the paper. The second author is partially supported by CNPq, Brazil, grant 303977/2015-9.

\section{References}

1. Albujer, A. L. and Alías, L. J., Spacelike hypersurfaces with constant mean curvature in the steady state space, Proc. Amer. Math. Soc. 137 (2009), 711721. MR2448594

2. Aledo, A., Romero, A. and Rubio, R., The existence and uniqueness of standard static splitting, Classical Quantum Gravity 32, 105004 (2015), 9 pp. MR3339851

3. Alías, L. J., Cánovas, V. and Colares, A. G., Marginally trapped submanifolds in generalized Robertson-Walker Spacetimes, Gen. Relativity Gravitation 49 (2017), 1-23. MR3599618

4. Anciaux, H., Marginally trapped submanifolds in space forms with arbitrary signature, Pacific J. Math. 272 (2014), 257-274. MR3284887

5. Anciaux, H. and Cipriani, N., Codimension two marginally trapped submanifolds in Robertson-Walker spacetimes, J. Geom. Phys. 88 (2015), 105-112. MR3293400

6. Anciaux, H. and Godoy, Y., Marginally trapped submanifolds in Lorentzian space forms and in the Lorentzian product of a space form by the real line, J. Math. Phys. 56, 023502 (2015), 12 pp. MR3390898

7. BAKRY, D. and ÉmerY, M., Diffusions hypercontractives, in Séminaire de probabilités, XIX, 1983/84, Lecture Notes in Math. 1123, pp. 177-206, Springer, Berlin, 1985. MR0889476

8. Beem, J., Ehrlich, P. and Easley, K. L., Global Lorentzian Geometry, 2nd ed., Pure and Applied Mathematics 202, Marcel Dekker, New York, 1996. MR1384756

9. Caminha, A., The geometry of closed conformal vector fields on Riemannian spaces, Bull. Braz. Math. Soc. (N.S.) 42 (2011), 277-300. MR2833803

10. Chen, B. Y., Classification of marginally trapped Lorentzian flat surfaces in $\mathbb{E}_{2}^{4}$ and its application to biharmonic surfaces, J. Math. Anal. Appl. 340 (2008), 861875. MR2390893

11. Chen, B. Y. and Van der Veken, J., Marginally trapped surfaces in Lorentzian space forms with positive relative nullity, Classical Quantum Gravity 24 (2007), 551563. MR2294231 
12. Chruściel, P. T., Galloway, G. J. and Ling, E., Weakly trapped surfaces in asymptotically de Sitter spacetimes, Classical Quantum Gravity 35, 135001 (2018), 9 pp. MR3826204

13. Corvino, J., Scalar curvature deformation and a gluing construction for the Einstein constraint equations, Comm. Math. Phys. 214 (2000), 137-189. MR1794269

14. Corvino, J. and Pollack, D., Scalar Curvature and the Einstein Constraint Equations, Surveys in Geometric Analysis and Relativity, ALM 20, pp. 145-188, International Press, Somerville, 2011. MR2906924

15. Corvino, J. and Schoen, R. M., On the asymptotics for the vacuum Einstein constraint equations, J. Differential Geom. 73 (2006), 185-217. MR2225517

16. Flores, J. L., Haesen, S. and Ortega, M., New examples of marginally trapped surfaces and tubes in warped spacetimes, Classical Quantum Gravity 27, 145021 (2010), 18 pp. MR2654079

17. Hawking, S. and Ellis, G., The large scale structure of space-time, Cambridge University Press, Cambridge, 1973. MR0424186

18. Hawking, S. W. and Penrose, R., The singularities of gravitational collapse and cosmology, Proc. R. Soc. Lond. Ser. A 314 (1970), 529. MR0264959

19. Kriele, M., Spacetime, Springer, Berlin, 1999. MR1726656

20. Mars, M. and Senovilla, J. M. M., Trapped surfaces and symmetries, Classical Quantum Gravity 20 (2003), 293-300. MR2025493

21. Miao, P. and TAm, L. F., On the volume functional of compact manifolds with boundary with constant scalar curvature, Calc. Var. Partial Differential Equations 36 (2009), 141-171. MR2546025

22. Omori, H., Isometric immersions of Riemannian manifolds, J. Math. Soc. Japan 19 (1983), 205-214. MR0215259

23. O'NeILl, B., Semi-Riemannian Geometry with Applications to Relativity, Academic Press, London, 1983. MR0719023

24. Penrose, R., Gravitational collapse and space-time singularities, Phys. Rev. Lett. 14 (1965), 57. MR0172678

25. Pigola, S., Rigoli, M. and Setti, A. G., Vanishing theorems on Riemannian manifolds, and geometric applications, J. Funct. Anal. 229 (2005), 424461. MR2182595

26. SÁnchez, M., Lorentzian manifolds admitting a killing vector field, Nonlinear Anal. 30 (1997), 643-654. MR1489831

27. SÁnchez, M., Geodesics in static spacetimes and $t$-periodic trajectories, Nonlinear Anal. 35 (1999), 677-686. MR1663623

28. SÁnchez, M., On the geometry of static spacetimes, Nonlinear Anal. 63 (2005), 45546.

29. SÁNCHEz, M., On causality and closed geodesics of compact Lorentzian manifolds and static spacetimes, Differential Geom. Appl. 24 (2006), 21-32. MR2193746

30. Senovilla, J. M. M., Singularity theorems and their consequences, Gen. Relativity Gravitation 30 (1998), 701-848. MR1623229

31. Senovilla, J. M. M., Trapped surfaces, horizons and exact solutions in higher dimensions, Classical Quantum Gravity 19, L113 (2002). MR1920305

32. Senovilla, J. M. M., Trapped submanifolds in Lorentzian geometry. Preprint (2005). arXiv : math/0412256v2. 
33. Troyanov, M., Parabolicity of Manifolds, Siberian Adv. Math. 9 (1999), 125150. MR1749853

34. Wei, G. and Willie, W., Comparison geometry for the Bakry-Émery Ricci tensor, J. Differential Geom. 83 (2009), 377-405. MR2577473

35. Yau, S. T., Harmonic functions on complete Riemannian manifolds, Comm. Pure Appl. Math. 28 (1975), 201-228. MR0431040

36. YAu, S. T., Some function-theoretic properties of complete Riemannian manifolds and their applications to geometry, Indiana Univ. Math. J. 25 (1976), 659670. MR0417452

Allan G. Freitas

Departamento de Matemática

Universidade Federal da Paraíba

58.051-900 João Pessoa

Paraíba

Brazil

allan@mat.ufpb.br

Henrique F. de Lima

Departamento de Matemática

Universidade Federal de Campina Grande

58.429-970 Campina Grande

Paraíba

Brazil

henrique@mat.ufcg.edu.br
Eraldo A. Lima Jr.

Departamento de Matemática

Universidade Federal da Paraíba

58.051-900 João Pessoa

Paraíba

Brazil

eraldo@mat.ufpb.br

Marcio S. Santos

Departamento de Matemática

Universidade Federal da Paraíba

58.051-900 João Pessoa

Paraíba

Brazil

marcio@mat.ufpb.br

Received February 26, 2019

in revised form May 28, 2019 